\title{
A Calibration Method of Multi-camera Pose Based on Monocular Vision
}

\author{
Dongzhao Huang ${ }^{\mathrm{a}, *}$, Qiancheng Zhao ${ }^{\mathrm{a}}$, Wenyun Wang ${ }^{\mathrm{a}}$, Tianlong Yang ${ }^{\mathrm{a}}$ \\ a'Hunan Provincial Key Laboratory of Health Maintenance for Mechanical Equipment, \\ Hunan University of Science and Technology, Xiangtan City, Hunan Province, China \\ *Corresponding Author: husthdz@163.com
}

\begin{abstract}
The detection of four-wheel alignment parameters has important impact on vehicle safety performance, and 3D image wheel aligner based on computer vision technology is the latest wheel alignment product. Extraction method for image feature of the target, and calibration method for the camera parameters associated with four-wheel alignment technology are often researched, but calibration method for multi-camera based on monocular vision is rarely researched. A convenient calibration method for multi-camera pose relationship is presented in this paper. The calibration device and calibration process for the method are all relatively simple. The device is a rigid-body consisting of two targets connected with a long beam, and the relative positional relationship between the two targets may be unknown before calibration. The results of simulation and experiment show that calibration method is feasible and effective, which can reach the calibration accuracy.
\end{abstract}

Keywords: Multi-camera Pose, Camera Calibration, Monocular Vision, Four-wheel Alignment.

\section{Introduction}

With the rapid growth in the number of vehicles, the quantity and quality of automotive testing equipment are constantly improving. As an important part of vehicle detection, detection of wheel alignment parameters has decisive impact on vehicle safety performance. If wheel alignment parameters are abnormal, it will result in abnormal wear of the tires, driving wandering, wheel shimmy, hard steering, the increment of fuel consumption and other issues. It directly affects driving safety of the automobile.

At present domestic and foreign automotive testing industry, the latest wheel alignment product is $3 \mathrm{D}$ image wheel aligner based on computer vision. The aligner is completely based on computer image processing technology, and it only needs two or more high-performance CCD cameras with four targets, without the traditional electronic sensors. It eliminates the circuit that may cause fault. Comparing with traditional 2D four-wheel aligner, it greatly reduces the number of sensors and doesn't need to be calibrated repeatedly. A single calibration of the parameters for the kind of aligner can be used repeatedly. The kind of aligner has the advantages such as simple operation, fast speed, high precision, etc.

Extraction method for image feature of the target ${ }^{(1-3)}$, calibration method for intrinsic parameters and external parameters of the camera ${ }^{(4,5)}$, calibration method for multi-camera pose relationship are the important parts of 3D image wheel alignment technology. Calibration method for multi-camera pose relationship based on binocular vision $^{(6,7)}$ is often researched, but calibration method for multi-camera pose relationship based on monocular vision is rarely researched. A convenient calibration method for multi-camera pose relationship based on monocular vision is presented in this paper.

\section{Calibration Method}

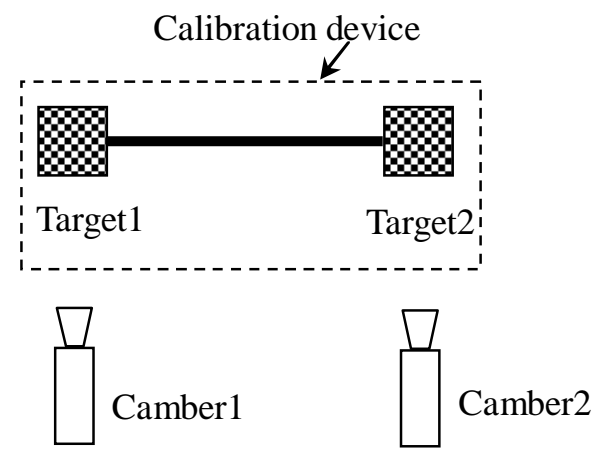

Fig. 1. Diagram for two-camera pose calibration 
Once the pose relationship of any two cameras is determined in the calibration of multi-camera, the pose relationship of multi-camera can be determined.

The model of two cameras pose calibration is shown in figure 1. The method presented in this paper needs a calibration device which is a rigid-body consisting of two targets connected with a long beam, and the relative positional relationship between the coordinate systems of the two targets may be unknown before calibration. When using the calibration device, it's necessary that camber 1 and camber 2 can take target 1 and target 2 respectively.

Assume the transformation matrix from the coordinate system of target 1 to the coordinate system of camber 1 is matrix $A$, the transformation matrix from the coordinate system of target 2 to the coordinate system of target 1 is matrix $M$, the transformation matrix from the coordinate system of camber 2 to the coordinate system of target 2 is matrix $B$, the transformation matrix from the coordinate system of camber 2 to the coordinate system of camber 1 is matrix $N$. Matrix $M$ and $N$ may be unknown before calibration, they can be solved after calibration.

According to single image, the relationship between matrixes $A, M, B$ and $N$ is shown as follows:

$$
\begin{aligned}
& N=A M B \\
& \text { And } N=\left[\begin{array}{llll}
n_{11} & n_{12} & n_{13} & n_{14} \\
n_{21} & n_{22} & n_{23} & n_{24} \\
n_{31} & n_{32} & n_{33} & n_{34} \\
0 & 0 & 0 & 1
\end{array}\right] \\
& A=\left[\begin{array}{llll}
a_{11} & a_{12} & a_{13} & a_{14} \\
a_{21} & a_{22} & a_{23} & a_{24} \\
a_{31} & a_{32} & a_{33} & a_{34} \\
0 & 0 & 0 & 1
\end{array}\right] \\
& M=\left[\begin{array}{llll}
m_{11} & m_{12} & m_{13} & m_{14} \\
m_{21} & m_{22} & m_{23} & m_{24} \\
m_{31} & m_{32} & m_{33} & m_{34} \\
0 & 0 & 0 & 1
\end{array}\right] \\
& B=\left[\begin{array}{llll}
b_{11} & b_{12} & b_{13} & b_{14} \\
b_{21} & b_{22} & b_{23} & b_{24} \\
b_{31} & b_{32} & b_{33} & b_{34} \\
0 & 0 & 0 & 1
\end{array}\right]
\end{aligned}
$$

Equation (1) can be transformed as follows:

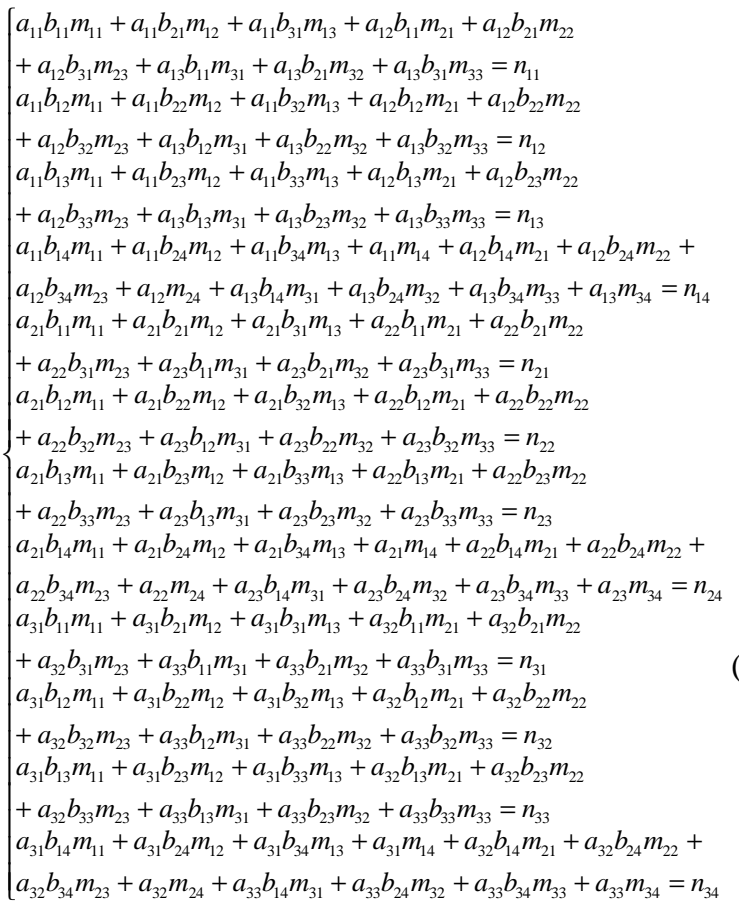

Equation group (6) can be transformed as equation (7):

$$
C D=E
$$

And $C=\left[\begin{array}{ll}C_{1} & C_{2}\end{array}\right]$

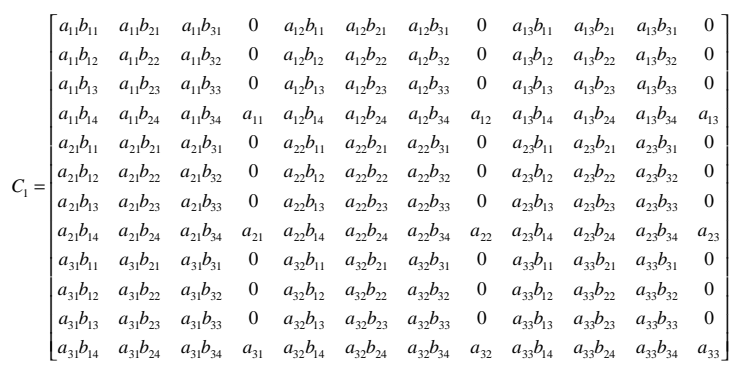

$C_{2}=\left[\begin{array}{cccccccccccc}-1 & 0 & 0 & 0 & 0 & 0 & 0 & 0 & 0 & 0 & 0 & 0 \\ 0 & -1 & 0 & 0 & 0 & 0 & 0 & 0 & 0 & 0 & 0 & 0 \\ 0 & 0 & -1 & 0 & 0 & 0 & 0 & 0 & 0 & 0 & 0 & 0 \\ 0 & 0 & 0 & -1 & 0 & 0 & 0 & 0 & 0 & 0 & 0 & 0 \\ 0 & 0 & 0 & 0 & -1 & 0 & 0 & 0 & 0 & 0 & 0 & 0 \\ 0 & 0 & 0 & 0 & 0 & -1 & 0 & 0 & 0 & 0 & 0 & 0 \\ 0 & 0 & 0 & 0 & 0 & 0 & -1 & 0 & 0 & 0 & 0 & 0 \\ 0 & 0 & 0 & 0 & 0 & 0 & 0 & -1 & 0 & 0 & 0 & 0 \\ 0 & 0 & 0 & 0 & 0 & 0 & 0 & 0 & -1 & 0 & 0 & 0 \\ 0 & 0 & 0 & 0 & 0 & 0 & 0 & 0 & 0 & -1 & 0 & 0 \\ 0 & 0 & 0 & 0 & 0 & 0 & 0 & 0 & 0 & 0 & -1 & 0 \\ 0 & 0 & 0 & 0 & 0 & 0 & 0 & 0 & 0 & 0 & 0 & -1\end{array}\right]$

$D=\left[\begin{array}{ll}D_{1} & D_{2}\end{array}\right]$

$D_{1}=\left[\begin{array}{llllllllllll}m_{11} & m_{12} & m_{13} & m_{14} & m_{21} & m_{22} & m_{23} & m_{24} & m_{31} & m_{32} & m_{33} & m_{34}\end{array}\right]$

$D_{2}=\left[\begin{array}{llllllllllll}n_{11} & n_{12} & n_{13} & n_{14} & n_{21} & n_{22} & n_{23} & n_{24} & n_{31} & n_{32} & n_{33} & n_{34}\end{array}\right]$

$E=\left[\begin{array}{llllllllllll}0 & 0 & 0 & -a_{14} & 0 & 0 & 0 & -a_{24} & 0 & 0 & 0 & -a_{34}\end{array}\right]^{T}$

1. The calibration of matrix $M:$ After the calibration 
device is placed at many positions and the corresponding images are taken, the transformation matrixes ( $A$ and $B$ ) for these positions can be gained. Based on these transformation matrixes, the equation (7) can be expanded as system of equations. At last, the transformation matrix $M$ can be calculated.

2. The calibration of matrix $N$ : When matrix $M$ is known, the matrix $N$ can be calibrated based on the image data at some positions.

The calculation method of matrix $N$ is shown as follows:

From equation (1), the follow equation can be gained:

$$
N B^{-1}=A M
$$

When matrix $M$ is known, the follow equation is gained:

$$
\begin{gathered}
F G=H \\
F=\left[\begin{array}{llllllllllll}
f_{11} & f_{21} & f_{31} & 0 & 0 & 0 & 0 & 0 & 0 & 0 & 0 & 0 \\
f_{12} & f_{22} & f_{32} & 0 & 0 & 0 & 0 & 0 & 0 & 0 & 0 & 0 \\
f_{13} & f_{23} & f_{33} & 0 & 0 & 0 & 0 & 0 & 0 & 0 & 0 & 0 \\
f_{14} & f_{24} & f_{34} & 1 & 0 & 0 & 0 & 0 & 0 & 0 & 0 & 0 \\
0 & 0 & 0 & 0 & f_{11} & f_{21} & f_{31} & 0 & 0 & 0 & 0 & 0 \\
0 & 0 & 0 & 0 & f_{12} & f_{22} & f_{32} & 0 & 0 & 0 & 0 & 0 \\
0 & 0 & 0 & 0 & f_{13} & f_{23} & f_{33} & 0 & 0 & 0 & 0 & 0 \\
0 & 0 & 0 & 0 & f_{14} & f_{24} & f_{34} & 1 & 0 & 0 & 0 & 0 \\
0 & 0 & 0 & 0 & 0 & 0 & 0 & 0 & f_{11} & f_{21} & f_{31} & 0 \\
0 & 0 & 0 & 0 & 0 & 0 & 0 & 0 & f_{12} & f_{22} & f_{32} & 0 \\
0 & 0 & 0 & 0 & 0 & 0 & 0 & 0 & f_{13} & f_{23} & f_{33} & 0 \\
0 & 0 & 0 & 0 & 0 & 0 & 0 & 0 & f_{14} & f_{24} & f_{34} & 1
\end{array}\right] \\
M_{f}=\left[\begin{array}{ccccccccc}
f_{11} & f_{12} & f_{13} & f_{14} \\
f_{21} & f_{22} & f_{23} & f_{24} \\
f_{31} & f_{32} & f_{33} & f_{34} \\
0 & 0 & 0 & 1
\end{array}\right] \\
0
\end{gathered}
$$

And $M_{f}$ is the inverse matrix of matrix $B$.

$$
G=\left[\begin{array}{llllllllllll}
n_{11} & n_{12} & n_{13} & n_{14} & n_{21} & n_{22} & n_{23} & n_{24} & n_{31} & n_{32} & n_{33} & n_{34}
\end{array}\right]^{T}
$$

$$
H=\left[\begin{array}{c}
a_{11} m_{11}+a_{12} m_{21}+a_{13} m_{31} \\
a_{11} m_{12}+a_{12} m_{22}+a_{13} m_{32} \\
a_{11} m_{13}+a_{12} m_{23}+a_{13} m_{33} \\
a_{11} m_{14}+a_{12} m_{24}+a_{13} m_{34}+a_{14} \\
a_{21} m_{11}+a_{22} m_{21}+a_{23} m_{31} \\
a_{21} m_{12}+a_{22} m_{22}+a_{23} m_{32} \\
a_{21} m_{13}+a_{22} m_{23}+a_{23} m_{33} \\
a_{21} m_{14}+a_{22} m_{24}+a_{23} m_{34}+a_{24} \\
a_{31} m_{11}+a_{32} m_{21}+a_{33} m_{31} \\
a_{31} m_{12}+a_{32} m_{22}+a_{33} m_{32} \\
a_{31} m_{13}+a_{32} m_{23}+a_{33} m_{33} \\
a_{31} m_{14}+a_{32} m_{24}+a_{33} m_{34}+a_{34}
\end{array}\right]
$$

When the device is placed at some positions and the corresponding images are taken, the transformation matrixes ( $A$ and $B$ ) for the positions can be gained. At last, the matrix $N$ can be gained based on equation (16).

\section{Simulation Result}

The transformation matrix can be expressed as follows:

$$
\begin{aligned}
& M_{R T}=\left[\begin{array}{cc}
R & T \\
0^{T} & 1
\end{array}\right] \\
& \text { Rotation matrix } R=\left[\begin{array}{lll}
r_{11} & r_{12} & r_{13} \\
r_{21} & r_{22} & r_{23} \\
r_{31} & r_{32} & r_{33}
\end{array}\right] \\
& \text { Translation matrix } T=\left[\begin{array}{lll}
T_{x} & T_{y} & T_{z}
\end{array}\right]^{T}
\end{aligned}
$$

In order to facilitate simulation, matrix $R$ will be expressed by means of Euler-angle representation as follows:

$$
\begin{aligned}
& R=R_{z}(\gamma) R_{y}(\beta) R_{x}(\alpha)=\left[\begin{array}{ccc}
\cos \gamma & -\sin \gamma & 0 \\
\sin \gamma & \cos \gamma & 0 \\
0 & 0 & 1
\end{array}\right] \\
& {\left[\begin{array}{ccc}
\cos \beta & 0 & \sin \beta \\
0 & 1 & 0 \\
-\sin \beta & 0 & \cos \beta
\end{array}\right]\left[\begin{array}{ccc}
1 & 0 & 0 \\
0 & \cos \alpha & -\sin \alpha \\
0 & \sin \alpha & \cos \alpha
\end{array}\right]} \\
& =\left[\begin{array}{ccc}
\cos \beta \cos \gamma & \sin \alpha \sin \beta \cos \gamma-\cos \alpha \sin \gamma & \cos \alpha \sin \beta \cos \gamma+\sin \alpha \sin \gamma \\
\cos \beta \sin \gamma & \sin \alpha \sin \beta \sin \gamma+\cos \alpha \cos \gamma & \cos \alpha \sin \beta \sin \gamma-\sin \alpha \cos \gamma \\
-\sin \beta & \sin \alpha \cos \beta & \cos \alpha \cos \beta
\end{array}\right]
\end{aligned}
$$

And $\beta=\arcsin \left(-r_{31}\right)$ 


$$
\begin{aligned}
& \alpha=\arcsin \left(\frac{r_{32}}{\cos \beta}\right) \\
& \gamma=\arcsin \left(\frac{r_{21}}{\cos \beta}\right)
\end{aligned}
$$

For matrix $M$ and matrix $N$, assume the values are all set as follows:

$$
\alpha=\beta=\gamma=T_{y}=T_{z}=0, T_{x}=2500
$$

The intrinsic parameters of camber 1 and camber 2 are all set as follows:

$$
\begin{aligned}
& \alpha_{x}=\alpha_{y}=3000, \gamma=k_{1}=k_{2}=p_{1}=p_{2}=0, \\
& u_{0}=1024, v_{0}=768
\end{aligned}
$$
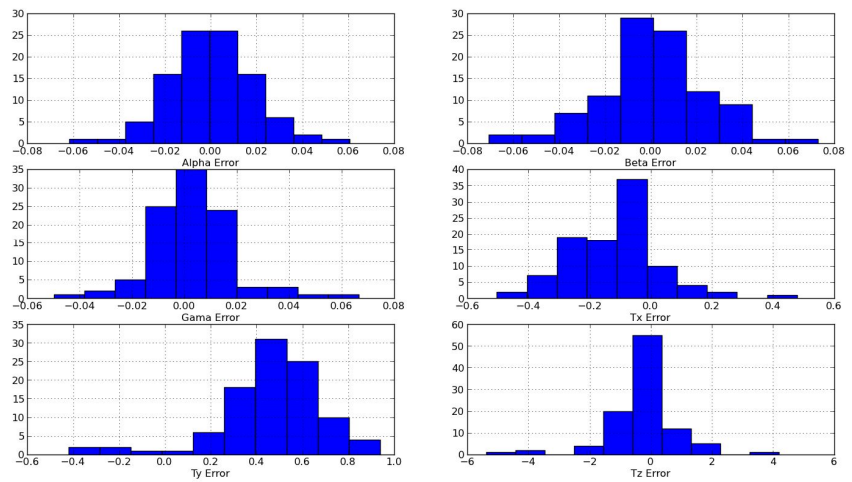

Fig. 2. Diagram for simulation result

When the extracted corner coordinates contains uniformly distributed white noise error of 0.1 pixel, the calibration error of matrix $N$ can be indirectly expressed by using the errors of $\alpha, \beta, \gamma, T_{x}, T_{y}$ and $T_{z}$. These errors are shown in figure 2, the units for the errors of $\alpha, \beta$ and $\gamma$ are all degrees, and the units for the errors of $T_{x}, T_{y}$ and $T_{z}$ are all millimeters.

\section{Experiment Result}

Based on the above calibration method and some other technology, a kind of new 3D four-wheel aligner is developed. Using this kind of 3D four-wheel aligner and another kind of 3D four-wheel aligner to detect a minibus respectively, the measurement results (only lists the toe-in angles of four wheels in this paper) are shown in table 1 and 2, respectively. The units of the angles in the two tables are all degrees.

Table 1. Result by using new aligner (unit: degree)

\begin{tabular}{|c|c|c|c|c|}
\hline No. & $\begin{array}{c}\text { left front } \\
\text { toe }\end{array}$ & $\begin{array}{c}\text { Right } \\
\text { front toe }\end{array}$ & $\begin{array}{c}\text { left behind } \\
\text { toe }\end{array}$ & $\begin{array}{c}\text { right behind } \\
\text { toe }\end{array}$ \\
\hline 1 & 0.08 & -0.01 & 0.1 & -0.2 \\
\hline 2 & 0.09 & -0.02 & 0.11 & -0.2 \\
\hline 3 & 0.12 & -0.04 & 0.1 & -0.2 \\
\hline 4 & 0.13 & -0.04 & 0.12 & -0.21 \\
\hline 5 & 0.1 & -0.03 & 0.1 & -0.19 \\
\hline
\end{tabular}

Table 2. Result by using another 3D aligner (unit: degree)

\begin{tabular}{|c|c|c|c|c|}
\hline No. & $\begin{array}{c}\text { left front } \\
\text { toe }\end{array}$ & $\begin{array}{c}\text { Right } \\
\text { front toe }\end{array}$ & $\begin{array}{c}\text { left behind } \\
\text { toe }\end{array}$ & $\begin{array}{c}\text { right behind } \\
\text { toe }\end{array}$ \\
\hline 1 & 0.07 & -0.05 & 0.09 & -0.19 \\
\hline 2 & 0.08 & -0.01 & 0.1 & -0.19 \\
\hline 3 & 0.14 & -0.06 & 0.09 & -0.17 \\
\hline 4 & 0.1 & -0.02 & 0.09 & -0.19 \\
\hline 5 & 0.06 & -0.04 & 0.12 & -0.21 \\
\hline
\end{tabular}

Comparing the data in the two tables, the results show that measurement stability and precision of the new 3D aligner are better than another kind of 3D aligner.

\section{Conclusions}

A calibration model of multi-camera pose relationship based on monocular vision is built. The calibration device and calibration process for the method are all relatively simple, and the results of simulation and experiment show that calibration method is feasible and effective. This method forms the foundation for four-wheel alignment technology based on computer vision theory. It's a new method that it is different from the traditional calibration method, and has broad application prospects.

\section{Acknowledgment}

The study is supported by the National Natural Science Foundation of China (51275169), Scientific Research Fund of Hunan Provincial Education Department (10C0684 and 12C0117), Science \& Research Programs of Hunan Province (2010TP4003-6 and 2012TP4023-4), Natural Science Foundation of Hunan Province (11JJ9016), Science \& Research Program of Hunan Province Key Projects (2011FJ2018 and 2012FJ4267), Aid program for Science 
and Technology Innovative Research Team in Higher Educational Institutions of Hunan Province.

\section{References}

(1) Jun-Sik Kim, and In-So Kweon. "Camera calibration based on arbitrary parallelograms", Computer Vision and Image Understanding, Vol. 113, pp. 1-10, 2009

(2) Pierre Gurdjos, Jun-Sik Kim, and In-So Kweon. "Euclidean structure from confocal conics: theory and application to camera calibration", Computer Vision and Image Understanding, Vol. 114, pp. 803-812, 2010

(3) Lars Krüger, and Christian Wöhler. "Accurate chequerboard corner localisation for camera calibration", Pattern Recognition Letters, Vol. 32, pp. 1428-1435, 2011

(4) Zhengyou Zhang. "A flexible new technique for camera calibration", IEEE Transaction on pattern analysis and machine intelligence, Vol. 22, No. 11, pp. 1330-1334, 2000

(5) Carlos Ricolfe-Viala, and Antonio-José Sánchez-Salmerón. "Using the camera pin-hole model restrictions to calibrate the lens distortion model", Optics \& Laser Technology, Vol. 43, pp. 996-1005, 2011

(6) Manuel E. Loaiza, Alberto B. Raposo, and Marcelo Gattass. "Multi-camera calibration based on an invariant pattern", Computers \& Graphics, Vol. 35, pp. 198-207, 2011

(7) Junhua Sun, Qianzhe Liu, Zhen Liu, and Guangjun Zhang. "A calibration method for stereo vision sensor with large FOV based on 1D targets", Optics and Lasers in Engineering, Vol. 49, pp. 1245-1250, 2011 